\title{
A subcutaneous implantable cardioverter-defibrillator (S-ICD) implantation in infection high-risk patient - a case study.
}

\author{
Michał Kowara ${ }^{1, B-E}$, Agnieszka Kołodzińska ${ }^{2, B-C, E}$, Marcin Grabowski ${ }^{3, A-F}$, Joachim Winter ${ }^{4, E}$, Grzegorz Opolski ${ }^{3, C, E}$ \\ A - Research concept and design, B - Collection and/or assembly of data, C - Data analysis and interpretation, \\ D - Writing the article, E - Critical revision of the article, F - Final approval of article \\ 1. 1st Department of Cardiology, Medical University of Warsaw, ul. Banacha 1a, 02-097 Warsaw, Department of Experimental and Clinical Physiology, \\ Medical University of Warsaw, ul. Banacha 1b, 02-097 Warsaw \\ 2. 1st Department of Cardiology, Medical University of Warsaw, ul. Banacha 1a, 02-097 Warsaw \\ 3. 1st Department of Cardiology, Medical University of Warsaw, ul. Banacha 1a, 02-097 Warsaw \\ 4. Division of Cardiac Surgery, University of Düsseldorf, Moorenstrasse 5, 40225 Düsseldorf, Germany.
}

Address for correspondence:

Michał Kowara, 1st Department of Cardiology, Medical University of Warsaw, ul. Banacha 1a, 02-097 Warsaw email: michal.kowara@wum.edu.pl Agnieszka Kołodzińska, 1st Department of Cardiology, Medical University of Warsaw, ul. Banacha 1a, 02-097 Warsaw
email: aa.kolodzinska@wp.pl

Marcin Grabowski, 1st Department of Cardiology, Medical University of Warsaw, ul. Banacha 1a, 02-097 Warsaw

email: grabowski.marcin@me.com

Joachim Winter, Division of Cardiac Surgery, University of Düsseldorf, Moorenstrasse 5, 40225 Düsseldorf, Germany. email: wintergina@web.de

Grzegorz Opolski, 1st Department of Cardiology, Medical University of Warsaw, ul. Banacha 1a, 02-097 Warsaw email: grzegorz.opolski@wum.edu.pl

Received: 01.10 .2017

Revised: 11.11 .2017

Accepted: 21.11 .2017

DOI: ???????????????????????

\section{Key words:}

transvenous lead extraction, subcutaneous implantable cardioverter-defibrillator, lead-related infective endocarditis

\begin{abstract}
The cardiac device related infective endocarditis (CDRIE) is an important indication for the implantable cardiac device extraction. In patients after recovery from CDRIE, who present indication for implantable cardioverter-defibrilator (ICD) but without necessity for pacing therapy the subcutaneous ICD (S-ICD) seems to be a reasonable alternative. Here we present a patient with CRT-D device transferred to our hospital with
\end{abstract}

\section{Case description}

The male patient of age 61 with a CRT-D device implanted 6 months earlier due to systolic heart failure was transferred from a local district hospital, with suspicion of cardiac device related infective endocarditis (CDRIE). At admission, the patient presented with fever $\left(38.5^{\circ} \mathrm{C}\right)$, leukocytosis $(\mathrm{WBC}$ 13.5x103/mm3), and increased CRP (334 mg/l). Blood culture the diagnosis of CDRIE and many comorbidities. The patient presented increased inflammatory markers serum level during the entire period of hospitalization, after device explanation and termination of antibiotic therapy. The blood culture tests were negative and transesophageal echocardiography showed no sign of endocarditis, therefore despite inflammatory biomarkers the patient has been implanted with S-ICD.

tests were positive for Gram-positive cocci. Transesophageal echocardiography revealed lead-attached vegetations. On the basis of this signs, the diagnosis of cardiac device related infective endocarditis (CDRIE) was confirmed. In addition, the patient presented with other comorbidities: diabetes mellitus with diabetic foot syndrome, persistent atrial fibrillation, coronary artery disease, chronic renal failure of level G3a, obesity 
and dental caries. Moreover, left subclavian vein thrombosis was also diagnosed on the basis of ultrasonography performed due to left upper extremity edema on physical examination. At admission, antibiotic therapy was introduced with vancomycin, rifampicin and gentamicin, whereas the infected CRT-D device was extracted after 4 weeks. Antibiotic therapy with vancomycin was ceased 6 weeks after the lead extraction (therapy with gentamicin - after two weeks). Blood culture tests were negative. Subclavian vein thrombosis also disappeared after one month of therapy. Diabetic foot syndrome was treated under the care of a specialist in a diabetology unit and dental caries was treated with teeth extraction by a maxillofacial surgeon. During the course of therapy, renal failure was exacerbated and the glomerular filtration rate decreased from $60-50$ to $22.4 \mathrm{ml} / \mathrm{min}$. When the antibiotic therapy was finished, the patient's general condition ameliorated, the glomerular filtration rate (GFR) level normalized, there were no clinical signs of infection, yet CRP levels were still high $(170 \mathrm{mg} / \mathrm{ml})$ with a normal procalcitonin level. In transesophageal echocardiography there was no presence of vegetation. In transthoracic echocardiography left ventricle ejection fraction was low $-30 \%$. Before CDRIE the patient had been implanted with a CRT-D due to heart failure with decreased ejection fraction (EF) and left bundle branch block (LBBB) in ECG shown in Figure 1 - ESC guidelines, class IIa ${ }^{[1]}$.
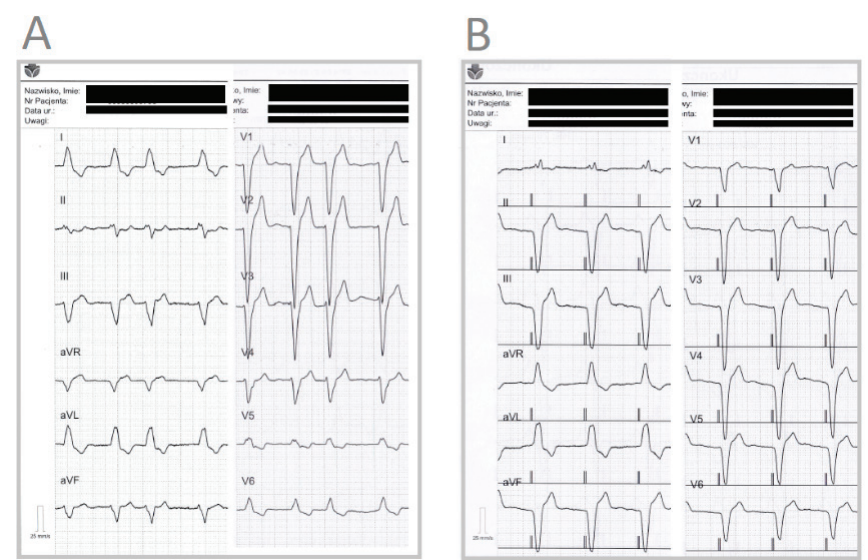

Figure 1. ECG before (A) and after (B) CRT-D implantation in the patient

The risk of infection as well as CDRIE recurrence was very high. Although an implantable cardioverter-defibrillator (ICD) was still recommended (class I) in the patient ${ }^{[1]}$, the decision of transvenous ICD system implantation was considered too risky. In this situation the novel method of a subcutaneous ICD (S-ICD) system implantation is a reasonable alternative, especially because there was no indication for pacing therapy in the patient (no episodes of bradyarrhythmia under telemetric control) - class IIa according to ESC guidelines ${ }^{[2]}$. Finally, the S-ICD system was implanted - the device was placed on the left anterior axillary line (Figure 2).

There were no perioperative complications. The position of the device and lead was checked intraoperatively and post-operatively by X-ray (Fig. 3). The Defibrillation Threshold Testing (DFT) was performed intraoperatively and the cardioverter-defibrillator was initially set up (Fig. 4).
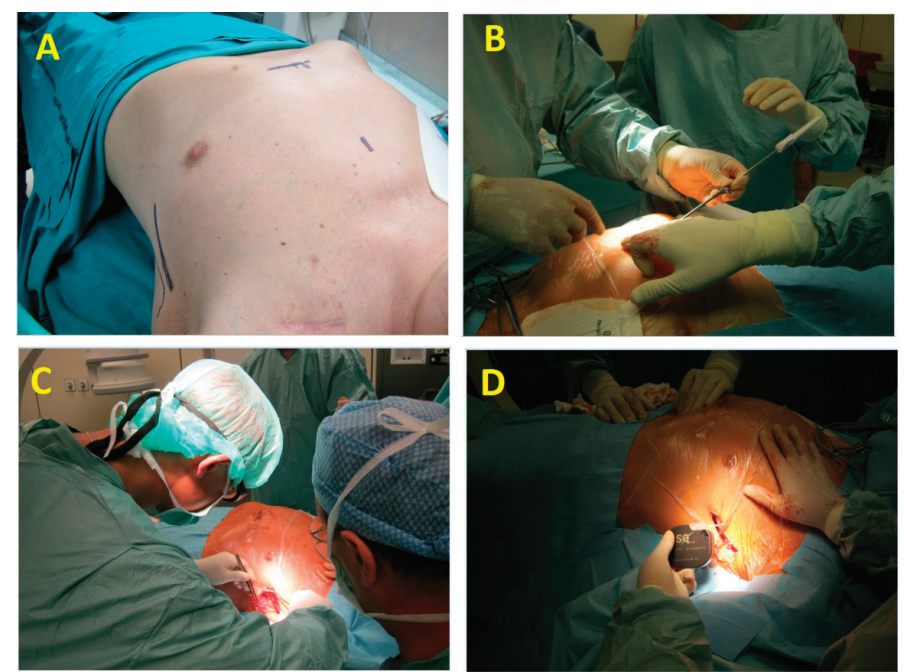

Figure 2. An S-ICD implantation by steps: A - site marking, B - lead positioning, C - skin incision, $\mathrm{D}$ - device implantation.

In the following days, the inflammatory markers decreased (CRP to $70 \mathrm{mg} / \mathrm{dl}$ ) and the patient was discharged in a good general status. Regular follow-up visits in the Implantable Cardiac Device Control and Telemonitoring Workshop, 1st Department of Cardiology were scheduled. To date, no complications have been reported.
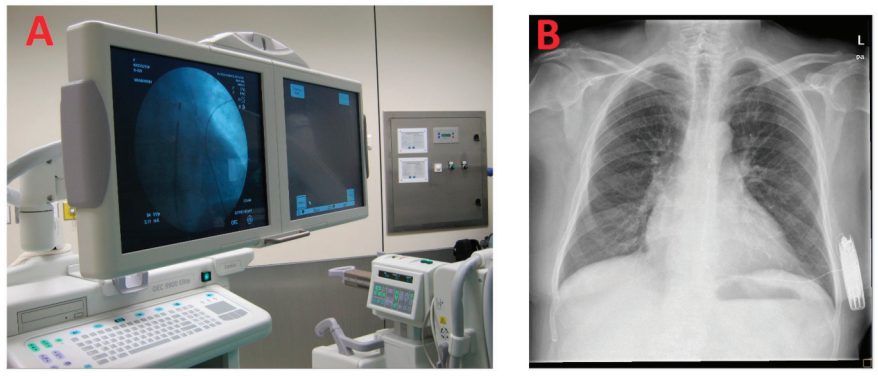

Figure 3. Assessment of S-ICD (device and lead) position by X-ray: A - intraoperatively, $\mathrm{B}$ - post-operatively (chest $\mathrm{X}$-ray, posteroanterior projection).

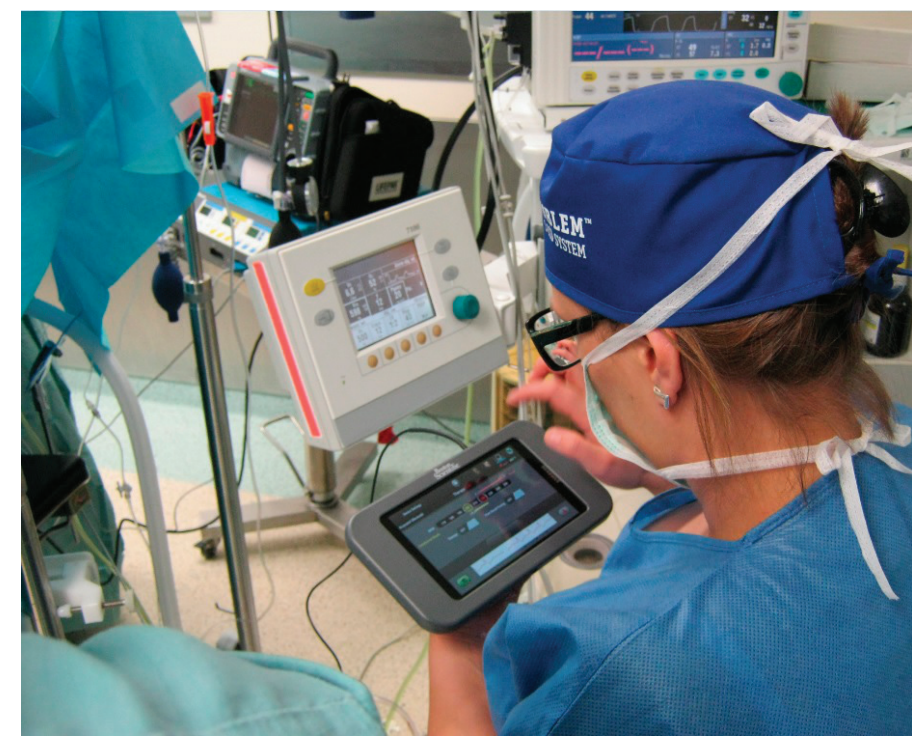

Figure 4. Initial program set-up for S-ICD performed directly after implantation. 


\section{Comments}

Cardiac device related infective endocarditis is an indication for removal of the entire cardiac device hardware, according to ESC guidelines ${ }^{[3]}$. However, chronic heart failure with decreased LVEF $<35 \%$ is an indication for ICD implantation ${ }^{[1]}$. The indications for reimplantation should be reassessed with caution in a patient after CDRIE and the optimal time for reimplantation remains controversial. Repeated procedures are independent risk factors for device-related infections according to multivariate analysis ${ }^{[4]}$. Diabetes and underlying heart disease are also considered as independent risk factors for infection of implantable cardiac devices ${ }^{[5]}$. Moreover, a patient after ICD implantation is more prone to infection than a patient after pacemaker implantation ${ }^{[6]}$. Our patient presented all the risk factors mentioned above. In addition, he presented with an increased CRP level even after completion of antibiotic therapy. Nowadays, the S-ICD is a reasonable alternative for this group of patients, with respect to absolute absence of bradyarrhythmic indications for pacing ${ }^{[7-9]}$. Moreover, the aforementioned device is more appropriate for high infection risk patients, because its lead is localized parallel to the parasternal line and does not contact the heart chambers, so the risk of cardiovascular implantable electronic device (CIED) infection is decreased ${ }^{[8,10]}$. The systematic review performed by Chue et al. showed that only $2.7 \%$ of subjects after S-ICD implantation developed pocket infection in short-term observation $^{[11]}$.

\section{Acknowledgements}

We would also like to acknowledge Marcin Michalak, M.D., $\mathrm{PhD}$ and Andrzej Cacko, M.D., PhD from the 1st Department of Cardiology.

\section{Disclosures}

None declared.

\section{References}

1. Ponikowski P, Voors AA, Anker SD, Bueno H, Cleland JG, Coats AJ, Falk V, González-Juanatey JR, Harjola VP, Jankowska EA, Jessup M, Linde C, Nihoyannopoulos P, Parissis JT, Pieske B, Riley JP, Rosano GM, Ruilope LM, Ruschitzka F, Rutten FH, van der Meer P; Authors/ Task Force Members. 2016 ESC Guidelines for the diagnosis and treatment of acute and chronic heart failure: The Task Force for the diagnosis and treatment of acute and chronic heart failure of the European Society of Cardiology (ESC)Developed with the special contribution of the Heart Failure Association (HFA) of the ESC. Eur Heart J. 2016 Jul 14;37(27):2129-200.. Epub 2016 May 20.

2. Priori SG, Blomström-Lundqvist C, Mazzanti A, Blom N, Borggrefe M, Camm J, Elliott PM, Fitzsimons D, Hatala R, Hindricks G, Kirchhof P, Kjeldsen K, Kuck KH, Hernandez-Madrid A, Nikolaou N, Norekvål TM, Spaulding C, Van Veldhuisen DJ; Task Force for the Management of Patients with Ventricular Arrhyth- mias and the Prevention of Sudden Cardiac Death of the European Society of Cardiology (ESC). 2015 ESC Guidelines for the management of patients with ventricular arrhythmias and the prevention of sudden cardiac death: The Task Force for the Management of Patients with Ventricular Arrhythmias and the Prevention of Sudden Cardiac Death of the European Society of Cardiology (ESC) Endorsed by: Association for European Paediatric and Congenital Cardiology (AEPC). Europace. 2015 Nov;17(11):1601-87.

3. Authors/Task Force Members, Habib G, Lancellotti P, Antunes MJ, Bongiorni MG, Casalta JP, Del Zotti F, Dulgheru R, El Khoury G, Erba PA, Iung B, Miro JM, Mulder BJ, Plonska-Gosciniak E, Price S, Roos-Hesselink J, Snygg-Martin U, Thuny F, Tornos Mas P, Vilacosta I, Zamorano JL.2015 ESC Guidelines for the management of infective endocarditis: The Task Force for the Management of Infective Endocarditis of the European Society of Cardiology (ESC)Endorsed by: European Association for Cardio-Thoracic Surgery (EACTS), the European Association of Nuclear Medicine (EANM). Eur Heart J. 2015 Nov 21;36(44):3075-128.

4. Ann HW1, Ahn JY1, Jeon YD2, Jung IY2, Jeong SJ1, Joung B3, Lee M3, Ku NS1, Han SH1, Kim JM1, Choi JY4. Incidence of and risk factors for infectious complications in patients with cardiac device implantation. Int J Infect Dis. 2015 Jul;36:9-14. doi: 10.1016/j. ijid.2015.05.011. Epub 2015 May 14.

5. Hercé B1, Nazeyrollas P, Lesaffre F, Sandras R, Chabert JP, Martin A, Tassan-Mangina S, Bui HT, Metz D. Risk factors for infection of implantable cardiac devices: data from a registry of 2496 patients. Europace. 2013 Jan;15(1):66-70. doi: 10.1093/europace/eus284. Epub 2012 Oct 24.

6. Uslan DZ1, Sohail MR, St Sauver JL, Friedman PA, Hayes DL, Stoner SM, Wilson WR, Steckelberg JM, Baddour LM. Permanent pacemaker and implantable cardioverter defibrillator infection: a population-based study. Arch Intern Med. 2007 Apr 9;167(7):669-75.

7. Verma N1, Rhyner J, Knight BP. The subcutaneous implantable cardioverter and defibrillator: advantages, limitations and future directions. Expert Rev Cardiovasc Ther. 2015 Sep;13(9):989-99. doi: 10.1586/14779072.2015.1071189.

8. Burke MC1, Gold MR2, Knight BP3, Barr CS4, Theuns DA5, Boersma LV6, Knops RE7, Weiss R8, Leon AR9, Herre JM10, Husby M11, Stein KM11, Lambiase PD12. Safety and Efficacy of the Totally Subcutaneous Implantable Defibrillator: 2-Year Results From a Pooled Analysis of the IDE Study and EFFORTLESS Registry. J Am Coll Cardiol. 2015 Apr 28;65(16):1605-15. doi: 10.1016/j.jacc.2015.02.047.

9. Kowara M, Michalak M, Cacko A, Kołodzińska A, Welk E, Zawadzka-Byśko M, Opolski G, Grabowski $M$. First experience with a subcutaneous implantable cardioverter-defibrillator (S-ICD) implantation - a case 
series study of single Cardiology Center. Heart Beat 2016;1:18-23.DOI: https://doi.org/10.24255/hbj/67039

10. Kondo Y, Ueda M, Kobayashi Y, Schwab JO. New horizon for infection prevention technology and implantable device. J Arrhythm. 2016 Aug;32(4):297-302.

11. Chue CD, Kwok CS, Wong CW, Patwala A, Barker D, Zaidi A, Mamas MA, Cunnington C, Ahmed FZ. Efficacy and safety of the subcutaneous implantable cardioverter defibrillator: a systematic review. Heart. 2017 Sep;103(17):1315-1322. 